\title{
In-situ Compression Imaging of Polymer Foams using Synchrotron X-ray Computed Tomography
}

\author{
${ }^{1}$ Brian M. Patterson, ${ }^{2}$ Nikhilesh Chawla, ${ }^{2}$ Jason Williams, ${ }^{3}$ Xianghui Xiao, ${ }^{4}$ Mathew Robinson, \\ ${ }^{1}$ Zachary Smith, ${ }^{1}$ Kevin Henderson, ${ }^{1}$ Nikolaus Cordes \\ ${ }^{1}$ Materials Science and Technology Division, Los Alamos National Laboratory, Los Alamos, NM USA \\ ${ }^{2}$ Department of Materials Science and Engineering, School for Engineering of Matter, Transport, and \\ Energy, Arizona State University, Tempe, AZ \\ ${ }^{3}$ Advanced Photon Source, Argonne National Laboratory, Argonne, Illinois, USA \\ ${ }^{4}$ Atomic Weapons Establishment, Aldermaston, Reading, UK
}

Understanding the effects of a material's composition, geometry, aging, and processing upon the overall material performance requires a detailed understanding of their initial morphology and how the morphology changes under external stimuli. Using a laboratory based X-ray CT system, it is possible to image polymer foams as they are compressed, in an interrupted in-situ modality. Due to the low flux of a laboratory X-ray source, at least when compared to the synchrotron, stress relaxation must occur in the material before a tomogram can be collected, otherwise the residual sample motion leads to image blur. This relaxation process requires $\sim 20$ minutes, before the tomogram can be collected over $1^{+}$hours [1]. Important information regarding the compressive performance and morphology changes are lost during this stress relaxation (Figure 1, black box).

Third generation synchrotron light sources, such as the Advanced Photon Source, afford materials researchers unprecedented X-ray flux to help solve complex materials science challenges. Coupling this high flux with a high speed camera provides X-ray radiographs every millisecond and generates tomographic data in $\sim 1$ second. Adding a sample compression cell makes it possible to study the dynamic in-situ deformation of polymeric foams at a $10^{-2}$ strain rate.

In this study, synchrotron X-ray tomography at beamline 2-BM-A,B was used to capture the morphology changes in polymeric foam materials during dynamic compression. Twenty full tomograms of each sample were acquired in a washing machine motion while simultaneously compressing the samples up to $60 \%$. The materials studied included hydrogen blown silicone foam (LK3626), prilled urea silicone foam, syntactic Sylgard-184 and simple additively manufactured foams

With this technique, we can measure and correlate the differences in mechanical performance of polymeric foams to their physical/morphological changes. For example, LK3626 is a soft-structured hydrogen blown silicone foam (Figure 2). It has a has weak ligament structure which quickly proceeds directly buckling with almost no bending resistive force even at low compression (Figure 1, right) and compresses with no Poisson effect. Syntactic Sylgard 184 (Figure 3) on the other hand is a much stiffer material due to the reinforcement from the $100 \mu \mathrm{m}$ glass microballoons and exhibits a stress/strain curve with definitive bending/buckling/compressing regions (Figure 1, right). Acquiring the data continuously, with no pause for stress relaxation or pro-longed imaging times, allows for a much simpler mechanical interpretation via Gibson and Ashby.

\section{References:}

[1] BM Patterson, K Henderson, Z Smith (2012) Journal of Materials Science 48: 1986. 

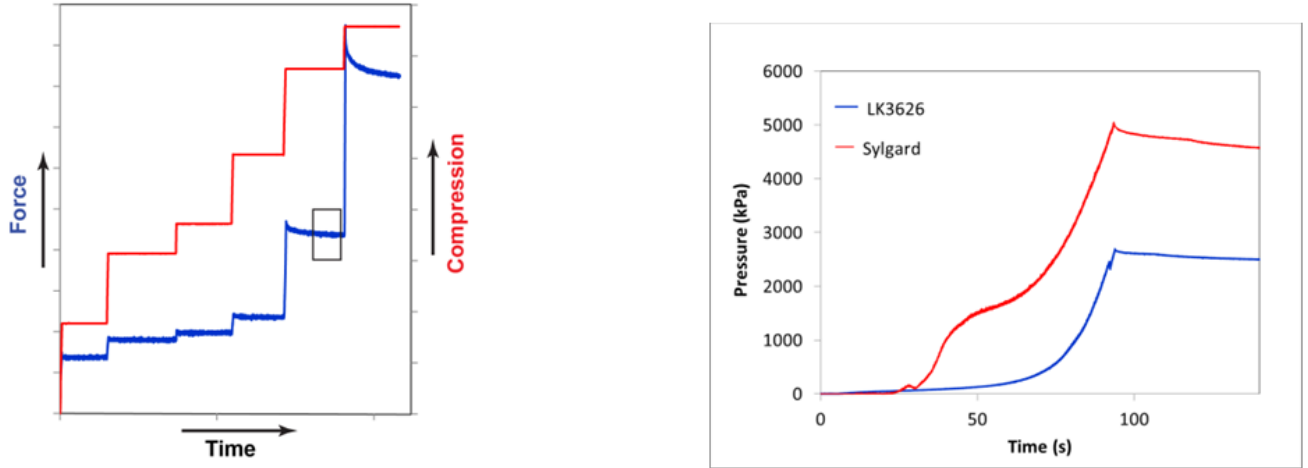

Figure 1: Typical interrupted in-situ force and compression curve for a foam in a laboratory setting (left). Laboratory systems must have stable samples and require a longer period of time to image. Imaging must occur after stress relaxation (black box). Force curves for two foams (right), LK3626 and syntactic Sylgard as they are continuously compressed at the synchrotron. Compression and imaging for these experiments halts at the maximum force at $\sim 100$ seconds.

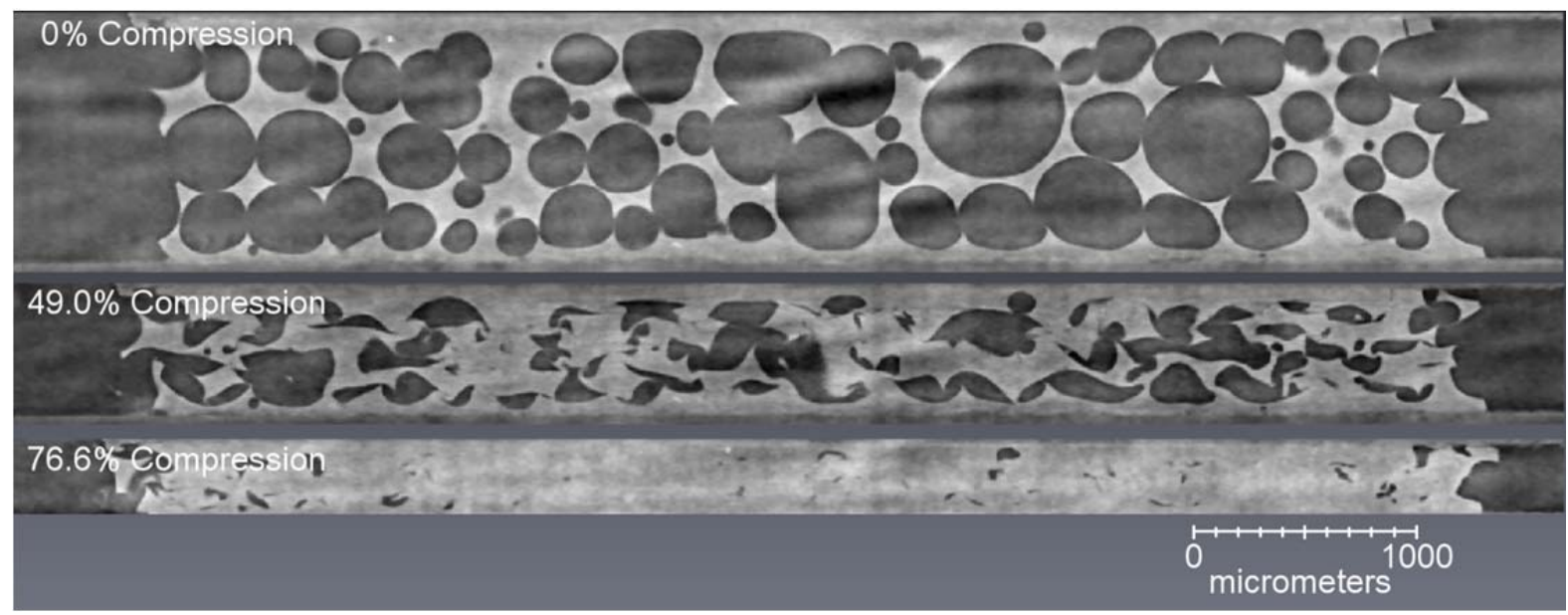

Figure 2: Compression of LK3626 hydrogen-blown foam. Sample is completely compressible with little Poisson effect. Ligaments can be seen breaking with material snapping back into place as it is compressed.

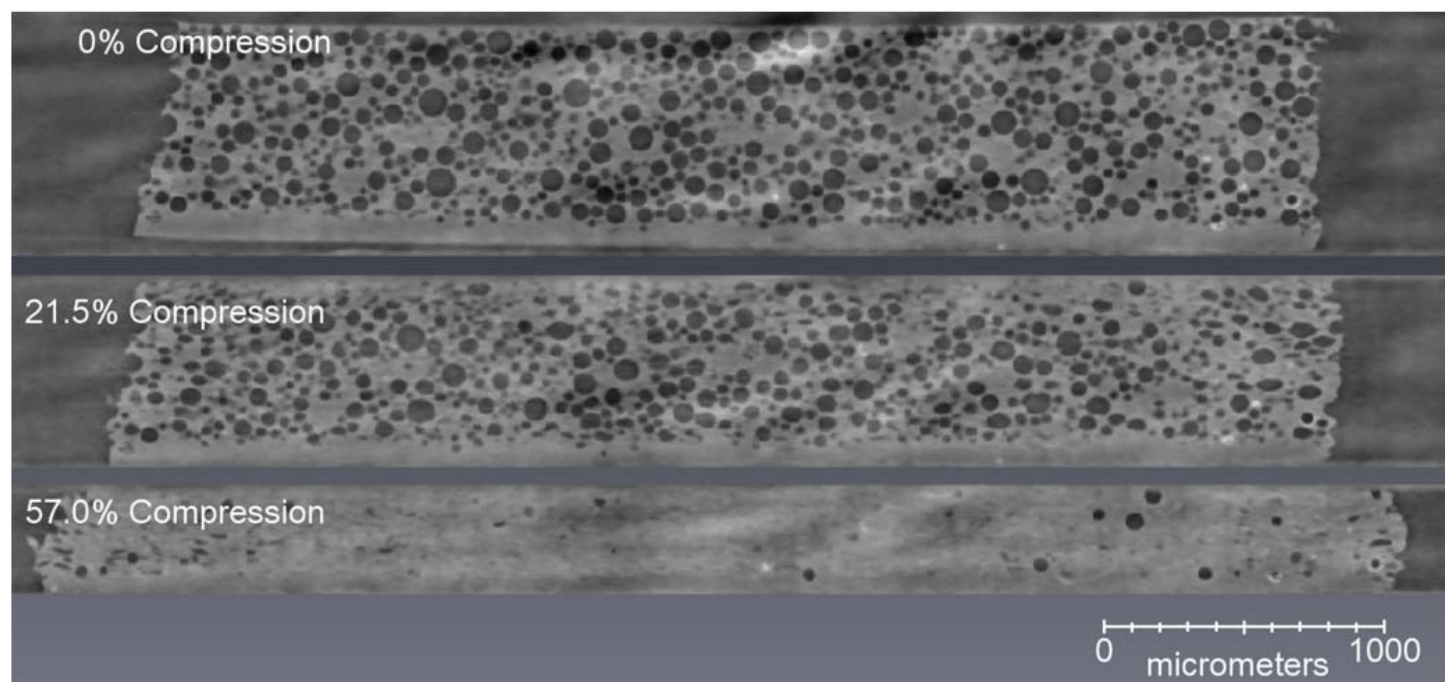

Figure 3: Compression of syntactic Sylgard foam with $100 \mu \mathrm{m}$ hollow glass beads. Sample is nearly incompressible with a high Poisson ratio. 